\title{
BMJ Open An exploration of counterfeit medicine surveillance strategies guided by geospatial analysis: lessons learned from counterfeit Avastin detection in the US drug supply chain
}

\author{
Raphael E Cuomo, ${ }^{1,2}$ Tim K Mackey ${ }^{1,3,4}$
}

To cite: Cuomo RE,

Mackey TK. An exploration of counterfeit medicine surveillance strategies guided by geospatial analysis: lessons learned from counterfeit Avastin detection in the US drug supply chain. BMJ Open 2014;4:e006657. doi:10.1136/bmjopen-2014006657

- Prepublication history and additional material is available. To view please visit the journal (http://dx.doi.org/ 10.1136/bmjopen-2014006657)

Received 17 September 2014 Revised 22 October 2014 Accepted 10 November 2014

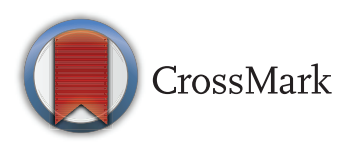

For numbered affiliations see end of article.

Correspondence to Professor Tim K Mackey; tmackey@ucsd.edu

\section{ABSTRACT}

Objective: To explore healthcare policy and system improvements that would more proactively respond to future penetration of counterfeit cancer medications in the USA drug supply chain using geospatial analysis.

Design: A statistical and geospatial analysis of areas that received notices from the Food and Drug Administration (FDA) about the possibility of counterfeit Avastin penetrating the US drug supply chain. Data from FDA warning notices were compared to data from 44 demographic variables available from the US Census Bureau via correlation, means testing and geospatial visualisation. Results were interpreted in light of existing literature in order to recommend improvements to surveillance of counterfeit medicines.

Setting/participants: This study analysed 791 distinct healthcare provider addresses that received FDA warning notices across 30431 zip codes in the USA.

Outcomes: Statistical outputs were Pearson's

correlation coefficients and $t$ values. Geospatial outputs were cartographic visualisations. These data were used to generate the overarching study outcome, which was a recommendation for a strategy for drug safety surveillance congruent with existing literature on counterfeit medication.

Results: Zip codes with greater numbers of individuals age $65+$ and greater numbers of ethnic white individuals were most correlated with receipt of a counterfeit Avastin notice. Geospatial visualisations designed in conjunction with statistical analysis of demographic variables appeared more capable of suggesting areas and populations that may be at risk for undetected counterfeit Avastin penetration.

Conclusions: This study suggests that dual incorporation of statistical and geospatial analysis in surveillance of counterfeit medicine may be helpful in guiding efforts to prevent, detect and visualise counterfeit medicines penetrations in the US drug supply chain and other settings. Importantly, the information generated by these analyses could be utilised to identify at-risk populations associated with demographic characteristics. Stakeholders should explore these results as another tool to improve on counterfeit medicine surveillance.
Strengths and limitations of this study

- This is the first study, to the best of our knowledge, to simultaneously use statistical analysis and geospatial modelling to analyse the distribution of counterfeit cancer medication warnings and their associated demographic risk characteristics.

- This article further strengthens this recommendation by suggesting that the designation of correlates a priori may be more useful to guide surveillance efforts when compared to intuitive designation of correlates a posteriori.

- Fundamentally, the results of this study may help to identify populations at risk, improve counterfeit drug surveillance, model future counterfeit medicine incidents, and communicate important drug safety information to the public and healthcare professionals.

- Our study relied on the use of data available from the Food and Drug Administration (FDA) that identifies US clinical practices that are suspected as being at risk for purchase or use of counterfeit Avastin. This data may be incomplete.

- There is no existing data that confirms how many patients were administered or otherwise received treatment with a counterfeit version of Avastin. Hence, our results and any potential conclusions on the impact of counterfeit Avastin are limited based on the data analysed. However, these data, to the best of our knowledge, are the most representative available compilation of addresses for potential counterfeit cancer medication exposure in the USA.

\section{INTRODUCTION}

In the USA, over 1.5 million people are expected to be diagnosed with cancer in 2014. ${ }^{1}$ A large number of these individuals will rely on pharmaceutical interventions in order to effectively treat their life-threatening conditions. However, an unknown number of these patients with cancer failed to receive effective 
treatment and may have suffered adverse consequences after becoming victims to a transnational criminal network profiting in the trade of counterfeit, substandard, adulterated and otherwise ineffective versions of cancer drugs. ${ }^{2-4}$ This patient safety failure was first uncovered in 2012 when the US Food and Drug Administration (FDA) announced it had detected counterfeit or fake versions of the injectable anticancer drug Avastin (INN: bevacizumab) in the US controlled drug supply chain. ${ }^{35}$

Avastin is an angiogenesis inhibitor that can be effective in treating several types of cancers, including those of the colorectum, lung, kidney and ovary. ${ }^{6-8}$ It is a highlyprescribed medication, and, in 2013, sales of Avastin reached over six billion dollars worldwide. ${ }^{9}$ Avastin is also a medication known to be counterfeited. ${ }^{10}{ }^{11}$ Though it is exclusively manufactured by only one authorised company in the USA (Genentech, a member company of Roche), counterfeit versions of Avastin containing no active pharmaceutical ingredient were purchased by hundreds of medical clinics in various US states from unauthorised domestic and foreign suppliers in violation of Federal law. ${ }^{5} 12$

Counterfeiting of medicines not only presents harms to individual health, but also poses problems for broader economic and social health-related outcomes. ${ }^{13-15}$ Despite these harms, current data available on counterfeit Avastin incidents are limited, making it is impossible to say with any certainty how many people received and were possibly administered counterfeit versions. Reports of counterfeit medicine detection are often based on journalistic discovery (as in the case of Avastin) ${ }^{16-18}$ and are therefore not the results of public health surveillance systems or adverse even reporting. Indeed, current global surveillance efforts are not sufficiently rigorous to provide a reliable estimate of overall counterfeit medicine prevalence needed to inform public health, drug regulatory or law enforcement activities. ${ }^{19} 20$

As an example, the only public safety information currently available on counterfeit Avastin incidents are warning letters issued by the FDA in two waves from 2012 to 2013 that were sent to approximately 1000 US clinical practices suspected of purchasing and/or administering counterfeit versions. ${ }^{3}{ }^{5} 21$ This occurred after the UK's Medicines and Healthcare Products Regulatory Agency prompted the FDA in December 2011 to examine the possibility of counterfeit Avastin entering the US drug supply chain. The FDA's subsequent investigation led to identification of US medical practices that had purchased multiple medications from certain unlicensed and foreign distributors that were also specifically identified as distributors of counterfeit Avastin. The FDA subsequently mailed by letter and posted on its website a warning letter to each of these identified practices (primarily consisting of business addresses for health clinics and individual physician practitioner recipients). These FDA warning letters are the only publicly available data identifying clinics/physicians who purchased and/or administered counterfeit Avastin, and the patient populations potentially impacted.
Collectively, limitations in available data point to a failure in public health surveillance for counterfeit medicines and the need to develop new methods aimed at generating critical data and information that stakeholders (including patients, healthcare providers, regulators, policymakers and law enforcement) need in order to monitor the occurrence, risk factors and safety implications of counterfeit drugs. ${ }^{22}{ }^{23}$ Hence, in this paper we describe a new methodology for assessing counterfeit drug safety warnings issued by the FDA, since they are the only data currently available. Our aims are to assess the geographic distribution of counterfeit Avastin warning notices in order to suggest information and methods that may be useful to incorporate into dynamic and proactive drug safety surveillance strategies for the future.

\section{METHODS}

Data on counterfeit Avastin notices were obtained from the FDA. ${ }^{24}$ They derive from two separate waves of distribution, first in 2012 (wave 1) and then in 2013 (wave 2). Data points from both waves were in the form of street addresses. From this data, a list of 791 unique zip codes were compiled where counterfeit Avastin notices had been sent. Three bivariate variables were created to designate zip codes where (1) a notice had been sent, (2) a notice had been sent in wave 1 and (3) a notice had been sent in wave 2 . Wave 1 notices originated from the FDA's original detection of counterfeit versions of Avastin from approximately nine drug distributors during 2012. Wave 2 notices originated from a second detection of counterfeit Avastin by FDA from a single distributor during 2013.

Three basemaps were downloaded from the US Census Bureau website for geospatial analysis: (1) 30431 US zip codes, and (2) 3233 US counties. ${ }^{25}$ The use of statistical results using data primarily at the zip code level were preferred over those at other levels (such as state level), as analyses at the zip code level carried a higher degree of resolution. However, in order to provide a more robust interpretation of analysis, we also adjusted our analysis to include the use of data at the county level as explained further.

In addition to geospatial parameters, the first basemap contained 44 demographic variables for nearly all zip codes. Analyses for this study included comparisons for areas of notice receipt versus areas of notice non-receipt, and also included comparisons for areas of wave 1 receipt versus areas of wave 2 receipt. Since the zip code-level basemap included demographic data for over 30000 spatial data points, this basemap was used to analyse whether these 44 demographic characteristics may have had a role in comparing these different sets of areas. These zip code-level characteristics were spatially amalgamated into counties for the purpose of producing maps. Therefore, the county-level basemap was primarily used for cartographic visualisation. A full list of variables is available in online supplementary appendix table 1 . 
To guide the construction of maps that compared demographics between notice receipt and notice nonreceipt, a table of pairwise correlations was made to determine which demographic variables were individually most correlated with zip codes having received a counterfeit Avastin notice. Means testing was not conducted, as the high number of non-receipt zip codes resulted in so many degrees of freedom that statistically significant differences would be detected in only slight differences between means. Common themes among most correlated variables were distinguished, and new variables were created as functions of the original 44 demographic variables. Most correlated variables were further checked at the county-level designation in order to determine whether or not these variables continued to be relatively highly correlated.

To guide the construction of maps that compared demographics between waves 1 and 2, t tests were conducted to compare means for demographic variables in zip codes where wave 1 notices were received and means for demographic variables in zip codes where wave 2 notices were received. Correlation statistics and means testing were computed using SPSS V.20 (IBM, Armonk, New York, USA).

All geospatial analyses were conducted with ArcGIS (ESRI, Redlands, California, USA). For a first set of maps, demographic variables most highly correlated with receipt of a counterfeit Avastin notice were used for geospatial analysis. For this initial set of maps, which used zip code-level designations, cut points for demographic variables that followed Poisson distributions were taken at the 85th and 98.5th centiles, in order to best avoid amalgamating multiple high levels in single categories. These variables were then displayed in choropleth maps of US zip codes, and the symbol of a circle was overlayed on top of zip codes where notices had been received. An additional map was created that displayed the geocoded locations of FDA-identified North American counterfeit Avastin distributors also with geocoded locations where FDA notices were received, in order to allow for visual inspection of potential relationships between these sets of addresses.

For a second set of maps, two point density maps were created to better visualise the frequency of warning notices and compare the differences in distribution of notices between waves. They included a map of wave 1 notices alone and another map for wave 2 notices alone. Twenty levels were chosen in a dark green-light green gradient in order to best display the difference between waves 1 and2. The Anselin Local Morans I statistic was subsequently calculated in order to produce a map, for a select demographic characteristic, that displayed a high-value cluster and low-value cluster, along with outliers within these clusters.

For a third set of maps, displays were made of the distributions for demographic variables of interest that exhibited significantly different means in zip codes receiving wave 1 notices compared to their means in zip codes receiving wave 2 notices. Demographic statistics for zip codes were amalgamated into counties (as a sum of zip codes) in order to better display national variations in a choropleth map. In order to further display such variations, two categories were created by dividing variable distributions by a single cut point which best allowed for the display of counties with relatively high levels of the identified demographic. Symbols representing waves 1 and 2 notices were then overlaid atop the choropleth demographic display in separate maps to assess differences in demographic risk characteristics between the waves.

\section{RESULTS}

The calculation of correlation coefficients showed that zip codes with many individuals in elderly age groups (over 65 years of age) were more correlated with the receipt of a counterfeit notice compared to zip codes with fewer elderly individuals. The number of individuals in a zip code racially self-identifying as white was also more correlated with the receipt of a notice (table 1). From these correlations it appears that the possibility of receiving a counterfeit Avastin notice may be related to age-related and race-related demographic distributions. A variable was then computed to amalgamate individuals in the top three age categorisations, thereby creating a variable representing the number of individuals over age 65. This new variable exhibited a Pearson's correlation coefficient $r$ of 0.260 . Variables most associated with the receipt of a counterfeit Avastin notice at the zip code level exhibited a much higher $r$ for certain demographics when analysis was adjusted to county-level data.

\begin{tabular}{|c|c|c|c|}
\hline \multicolumn{2}{|l|}{ Most correlated variables } & \multicolumn{2}{|l|}{ Least correlated variables } \\
\hline Variable & $\mathbf{r}$ & Variable & $\mathbf{r}$ \\
\hline Number of people age 85 and above & 0.263 & Average family size & 0.001 \\
\hline Number of people age 75-84 & 0.258 & Average household size & -0.002 \\
\hline Households with married couple and no children & 0.255 & Square miles & -0.011 \\
\hline Self-identifying individuals as racially white & 0.253 & $\begin{array}{l}\text { Self-identifying individuals as racially native } \\
\text { Hawaiian or Other Pacific Islander }\end{array}$ & 0.037 \\
\hline Number of people age $65-74$ & 0.252 & Median age of females & -0.037 \\
\hline
\end{tabular}



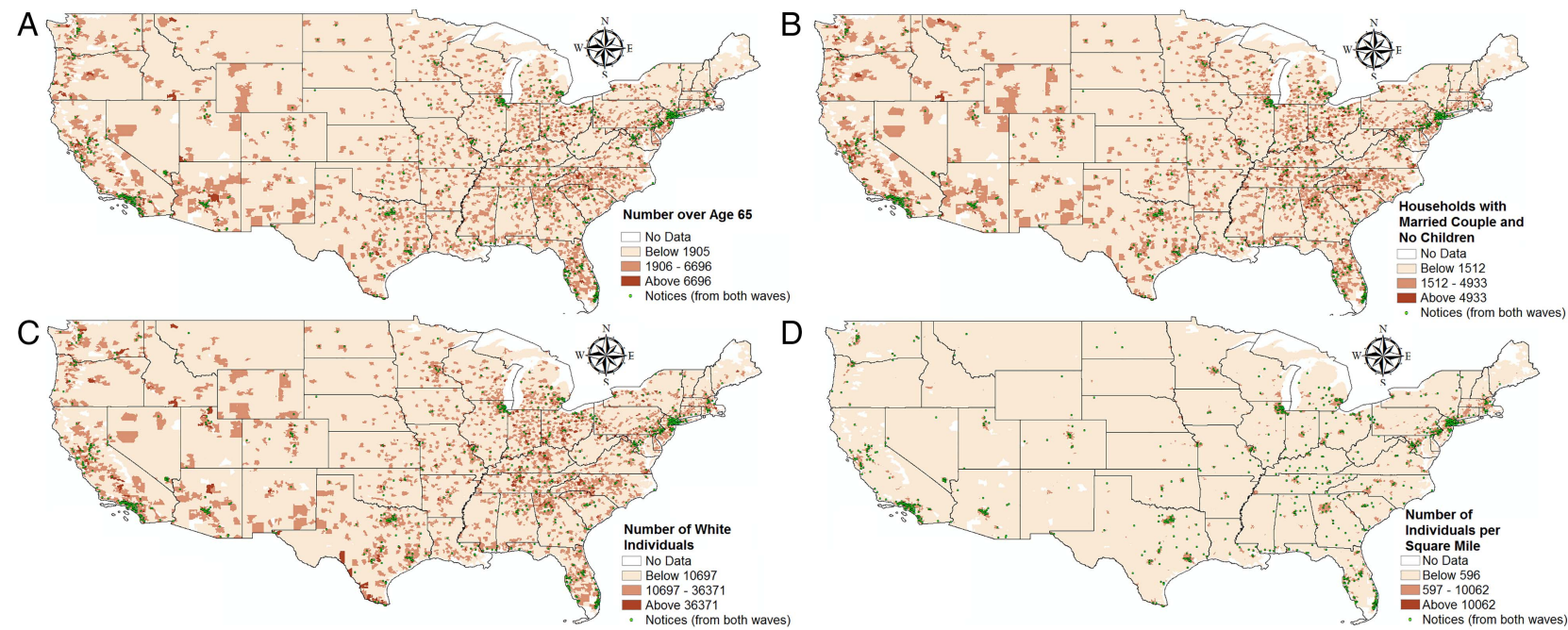

Figure 1 Choropleth maps of the USA with categorisations, at the zip code level, for demographic correlates of interest, specifically (A) number of individuals over age 65, (B) number of households with married couple and no children, (C) number of white individuals, and (D) number of individuals per square mile.

Specifically, for the number of people age 65 and above, $\mathrm{r}=0.922$; for the number of people self-identifying as racially white, $\mathrm{r}=0.936$; and for the number of households with married couples and no children, $\mathrm{r}=0.939$.

Geospatial analyses indicated that the more correlated demographic variables were typically in higher categories in zip codes that had received counterfeit notices (figure 1). The most correlated demographic variables followed Poisson distributions, so three categories were designated for these variables: below 85th centile, 85th to 98.5 th centile, and above 98.5 th centile. This analysis also revealed a notable number of zip codes that had not received counterfeit notices, despite having relatively higher categories of identified demographic correlates. When conducting mapping of addresses for North
American counterfeit distributors along with counterfeit notice recipients, visualisation revealed that some distributors appeared to be located among clusters of counterfeit notices (Southern California and New York), while other distributors did not appear to be located among clusters of counterfeit notices and were even located outside of the USA (ie, Canada; figure 2).

The comparison of a point density map for wave 1 notices to a point density map for wave 2 notices shows that wave 1 notices were more concentrated in Southern California (figure 3A), whereas wave 2 notices exhibited greater concentrations in the eastern half of the country (figure 3B). This indicates that the distribution of FDA warning notices was geographically distinct and needs to be analysed further for differences. A subsequent cluster

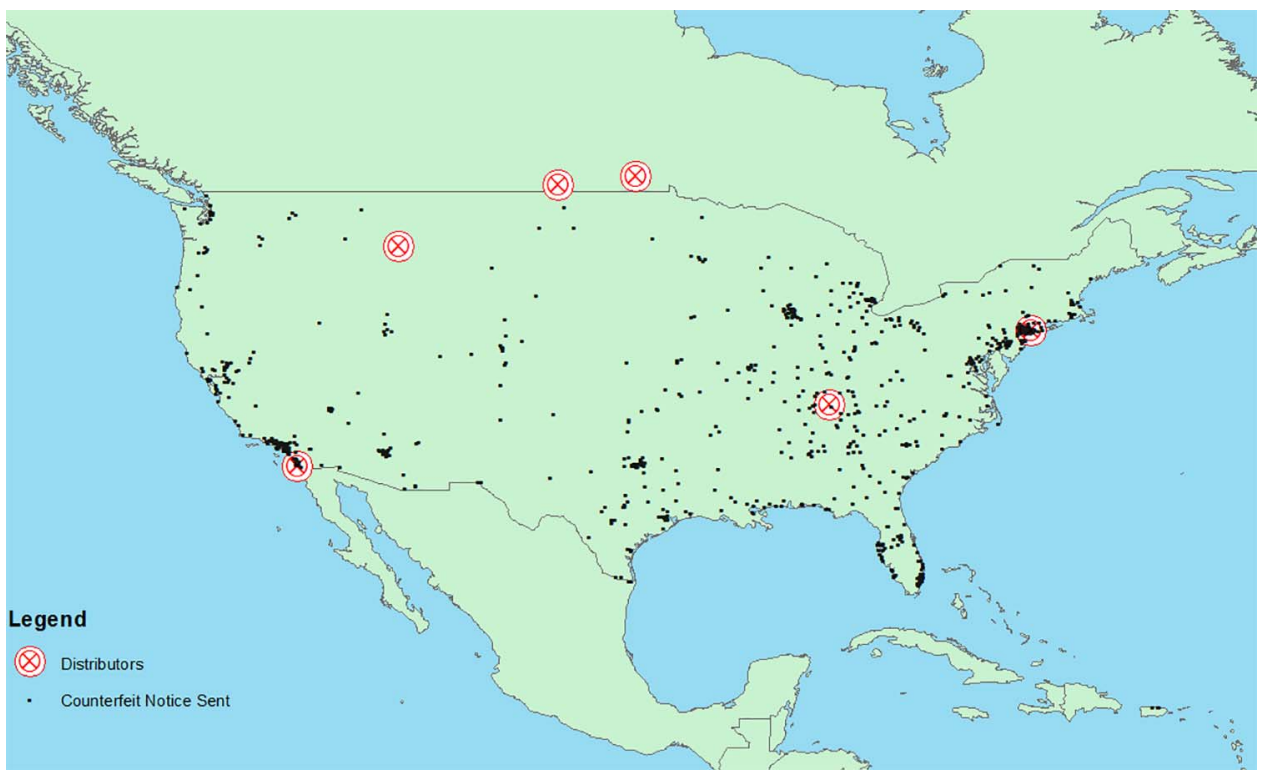

Figure 2 Map of North America showing locations of clinics receiving counterfeit notices along with locations of distributors. 
A

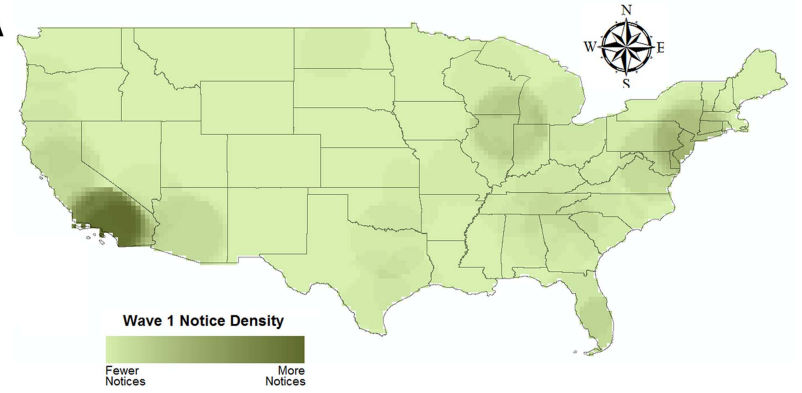

B

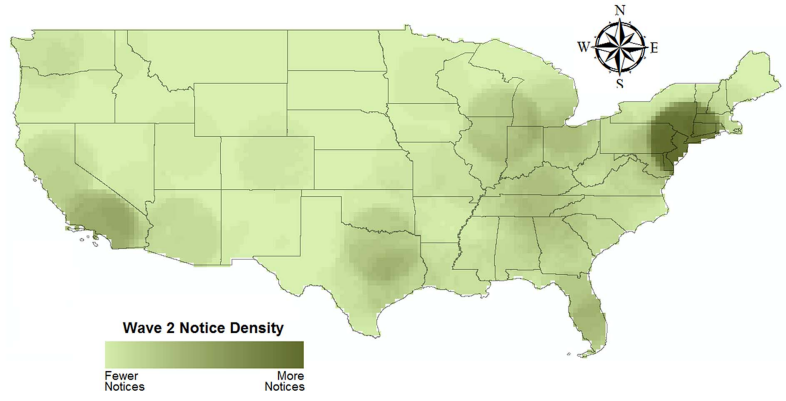

Figure 3 Point density maps of USA comparing distribution of Avastin warning notices for (A) wave 1 and (B) wave 2.

and outlier analysis showed clusters of high numbers of individuals over age 65 for metropolitan areas in Southern California, the New York metropolitan area, and the Miami metropolitan area (figure 4).

When examining in more detail the differences between waves 1 and $2, \mathrm{t}$ tests revealed four variables with significantly different means in wave 1 zip codes compared to means in wave 2 zip codes (table 2). Three of these variables pertained to race. Maps comparing counties with high numbers of multiracial individuals (above 2500 people) with the distributions of waves 1 and 2 notices revealed that many of these counties were in Southern California (figure 5), which is the same region where wave 1 notices were found to be concentrated in point density maps (figure $3 \mathrm{~A}$ ).

\section{DISCUSSION}

Several of the demographic correlates found in this study are consistent with demographic characteristics of areas with high numbers of patients with cancer. In particular, it was found that the number of elderly individuals (over age 65) in an area was more highly correlated with the receipt of a counterfeit Avastin notice. Accordingly, the median age of patients with cancer at diagnosis in the
USA is 66 years. ${ }^{26}$ Furthermore, it was also found that the number of white individuals was more highly correlated with the receipt of a counterfeit notice. In explaining this result we note that white individuals and white households hold greater annual incomes than the US average,${ }^{27}$ thereby making them more likely to afford healthcare coverage and giving them more access to expensive cancer medications such as Avastin. ${ }^{28}$

Figure 1 categorises demographic variables at the 85th and 98.5th centile thresholds in order to allow for comparison between variables determined prior to statistical analysis (ie, 'a priori') and after statistical analysis (ie, 'a posteriori'). These cut points were used because these variables were approximately distributed according to the Poisson distribution. Compared to figure 1D, an observation of figure $1 \mathrm{~A}-\mathrm{C}$ indicate that high levels of demographic correlates are evident along the northwest Interstate-84 highway, in parts of the Texas-Mexico border, the California Central Valley and other regions where very few cases have been detected. Figure 1A-C also suggest that distribution of counterfeit notices were lacking in the Piedmont Atlantic and Great Lakes megaregions, especially when compared to the high level of distributed notices in the Northeast, Florida and Southern California megaregions. Figure 1D appears much less suggestive of

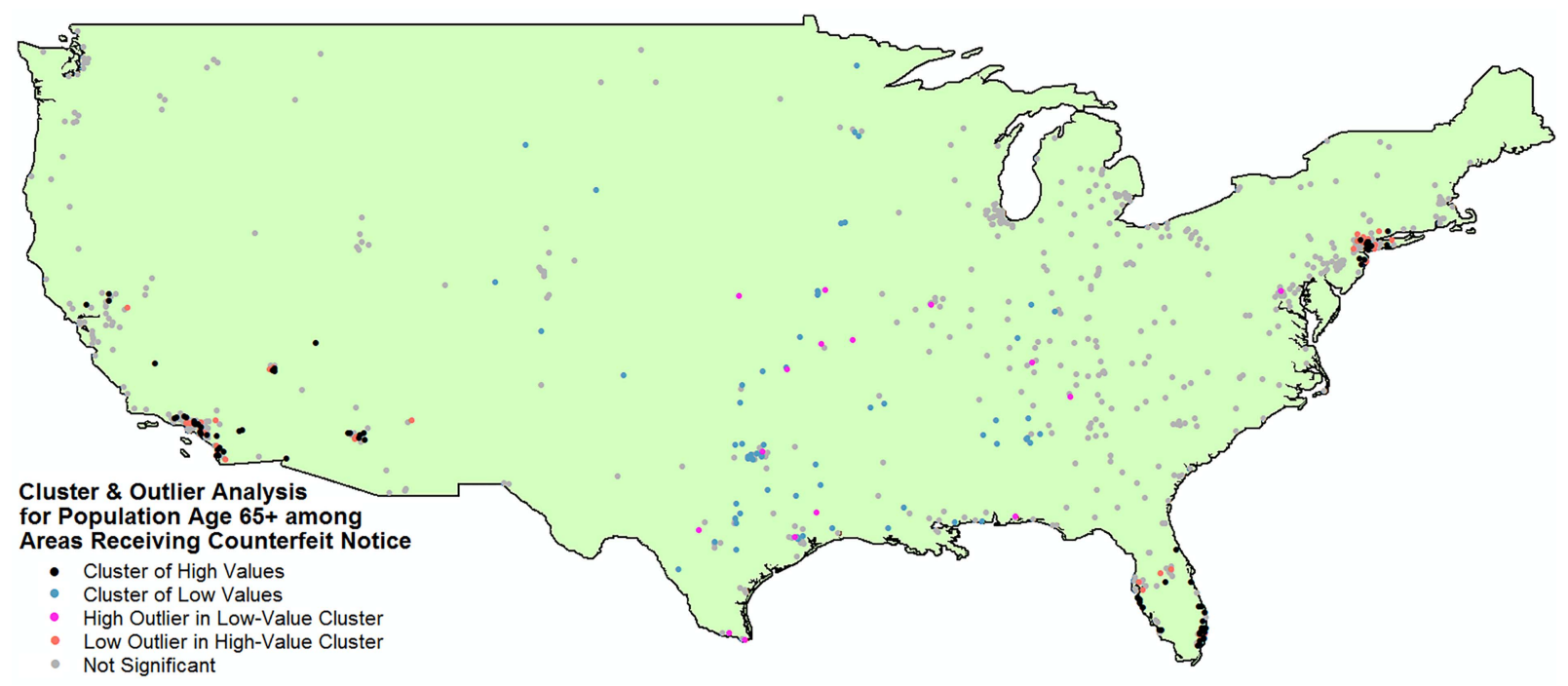

Figure 4 Analysis of clustering and outliers of clinics receiving warning notices, by number of individuals over age 65 . 
Table 2 Significant differences between notices sent in Wave 1 with those sent in Wave 2, by zip code $(n=871)$

\begin{tabular}{|c|c|c|c|}
\hline & \multicolumn{3}{|c|}{ Wave 1 vs wave $2^{*}$} \\
\hline & $\begin{array}{l}\text { Wave } 1 \\
(n=128) \\
\text { Mean }\end{array}$ & $\begin{array}{l}\text { Wave } 2 \\
(n=743) \\
\text { Mean }\end{array}$ & p Value \\
\hline Number of households having only Native Hawaiian and Other Pacific Islander & 102 & 47 & 0.045 \\
\hline Number of people with race other than white, black, Asian, or Hawaiian & 3553 & 1906 & 0.03 \\
\hline Number of people with two or more races & 1202 & 905 & 0.005 \\
\hline $\begin{array}{l}\text { Number of family Households, not married-couple family, male householder, } \\
\text { no wife present, with own children under } 18 \text { years }\end{array}$ & 357 & 294 & 0.024 \\
\hline
\end{tabular}

this disparity. Therefore, it appears that use of variables determined a posteriori are more likely than variables determined a priori to indicate geographic areas with undetected levels of counterfeit medicine. This conclusion suggests the added utility of using statistical analysis in conjunction with geospatial analysis, as opposed to only visually analysing geospatial output.

Figures 3 and 5 display differences detected between waves 1 and 2 distributions of counterfeit Avastin notices. Table 2 indicates that many of the statistically significant differences between the waves are associated with racial demographics. Though these predominantly racial demographics were found to significantly differ between waves 1 and 2 , these variables may not be explanatory for the receipt of a counterfeit Avastin notice, as they were not among the variables more highly correlated to the entire set of geographic areas where counterfeit notices were received. Therefore, these variables may be more useful in discerning differences between notice distributions, as opposed to explaining the geographic relationships related to counterfeit Avastin receipt. Figure 5 results seem to indicate that areas inhabited by many multiracial individuals are more closely related to the distribution of wave 1 notices than the distribution of wave 2 notices. These may be an artefact of higher frequency of warning letter distribution in certain regions with more diverse ethnic representation or may indicate a different sourcing pattern in wave 2 than in wave 1 . This potential shift in the possible at-risk patient population between the waves indicates the need for further study.
Though limited in their generalisability, the results from this study could be useful for the detection of at-risk populations and counterfeit drug penetrations of other cancer angiogenesis inhibitor class of drugs (eg, Nevaxar, Sutent, Votrient and Afinitor), since patients being prescribed those drugs may have similar demographic characteristics as those being prescribed Avastin. Indeed, legal documents associated with the detection of counterfeit Avastin have revealed that other cancer drugs were subject to counterfeiting and have also been distributed in the US drug supply chain though have yet to be studied. ${ }^{29} 30$

Overall, the general lack of analysis and validated data on counterfeit Avastin incidents raises key concerns. Primarily, though the FDA sent notices directly to clinics suspected of purchasing counterfeit Avastin, patients that may have been directly adversely impacted were not notified with the exception of a public service announcement posted on the FDA website providing general safety information. ${ }^{35}$ Additionally, legal prosecutions of clinics that received warning notices uncovered that they knowingly purchased counterfeit Avastin and continued its use even after nurse employees raised serious safety concerns, bringing into question the effectiveness of notices focused on clinical sites. ${ }^{31}$ Finally, lack of data limits efforts to estimate the true scope and impact of counterfeit Avastin cases (eg, the number of patients potentially impacted), including possible differences in the population and safety characteristics between warning notices in waves 1 and 2 that we have attempted to identify in this study. Collectively, these limitations hamper efforts to

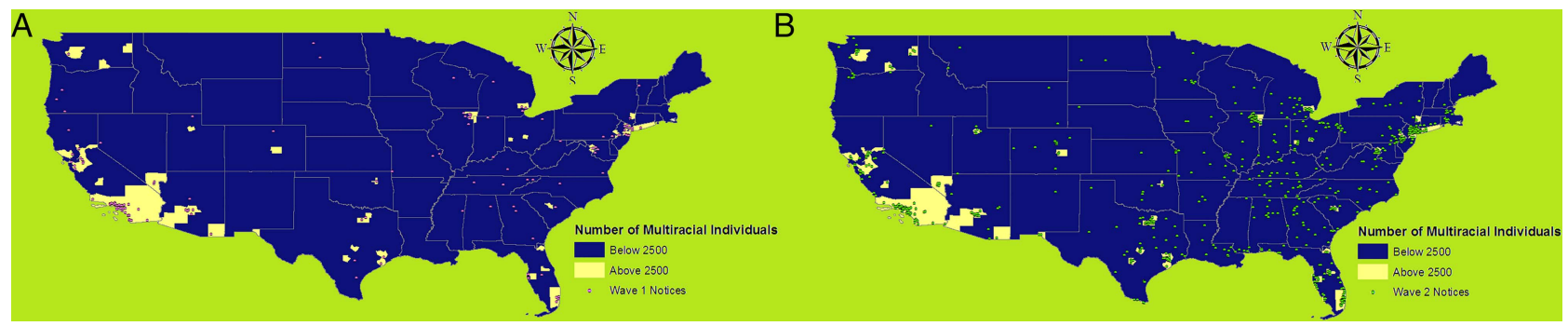

Figure 5 Choropleth maps of the USA comparing counties with greater than 2500 multiracial individuals with (A) Wave 1 notices and (B) Wave 2 notices. 
develop prevention strategies for counterfeit medicine incidents that may occur in the future.

Finally, the inadequacy of existing surveillance mechanisms and their associated data collection may in part relate to a lack of engagement with healthcare professionals (HCPs) on the issue. Though counterfeit versions of Avastin were easily detectable (ie, had foreign language markings on packaging/identified incorrect manufacturer), surveillance and reporting by HCPs was largely absent. A model that has the potential to proactively respond to the penetration of counterfeit Avastin could be one that encourages or requires the participation of HCPs in active surveillance for counterfeit and unapproved medications. Central to this type of model is education to HCPs on the patient safety and associated legal risks to the practitioner of sourcing and administering counterfeit medications, information on how to avoid such risks and providing better information on specific populations/areas of the country at-risk. If HCPs had greater awareness regarding sourcing risks and better information to adequately detect counterfeit versions, they may have acted as important data sources for prevention, surveillance and reporting. Such reporting is currently possible and indeed required in the FDA Adverse Event Reporting System (FAERS), but would likely be enhanced through education, collaborations, and partnerships on drug safety and surveillance strategies for counterfeit medicines. ${ }^{32} 33$

\section{Limitations}

Our study relied on the use of data available from the FDA that identifies US clinical practices that are suspected as being at risk for purchase or use of counterfeit Avastin. However, this data may be incomplete. As an example, some demographic variables we identified as being associated with receipt of a counterfeit Avastin notice may simply reflect the geographic distribution of demographic variables associated with Avastin-treatable cancers. Analysis of data more accurately describing the geographic distribution of Avastin (ie, prescribing and/ or reimbursement data) and US cancer incidence/ prevalence data has the potential to address this limitation, but was beyond the scope of this study. Further, there is no data that confirms how many patients were administered or otherwise received treatment with a counterfeit version of Avastin. Hence, our results and any potential conclusions on the impact of counterfeit Avastin are limited based on the data analysed. Despite these limitations, these data, to the best our knowledge, are the most representative available compilation of addresses for potential counterfeit cancer medication exposure in the USA.

The demographic variables analysed in this study were those made publically available along with geospatial data packaged for analysis through the use of geospatial software. Though these 44 characteristics provide a preliminary understanding of demographic differences by counterfeit notice receipt status, possibilities for redundancy and incompleteness exist. For example, the variables that represent the number of individuals within certain age groups may serve as a proxy measure for susceptibility to several cancers that are treatable with Avastin, thereby increasing the possibility that this age group is more highly prescribed Avastin, which in turn increases the possibility that they went to a clinic that purchased and administered a counterfeit version. A similar limitation may apply to the variable of individuals over age 65 , as these individuals may have greater access to healthcare services/coverage through their eligibility for enrolment into Medicare, resulting in higher levels of access to Avastin treatment. Given the high price of Avastin, a measure of health insurance coverage might also be confounded by income, as income may also equate to increased treatment access through additional enrolment into a supplemental Medicare insurance programme. Therefore, while measures of income and insurance coverage were omitted from this study, further exploration of the complex multivariate relationships between Avastin financing, economics and access are needed to further validate study results.

\section{Strengths}

This is the first study, to the best our knowledge, to simultaneously use statistical analysis and geospatial modelling to analyse the distribution of counterfeit cancer medication and their associated demographic risk characteristics. This article further strengthens this recommendation by suggesting that the designation of correlates a priori may be more useful to guide surveillance efforts when compared to intuitive designation of correlates a posteriori. Fundamentally, the results of this study may help to identify populations at risk, improve counterfeit drug surveillance, model future counterfeit medicine incidents notification and communicate important drug safety information to the public and HCPs.

\section{CONCLUSION}

In this study, we explore new methods and strategies to better assess the distribution of counterfeit cancer medicine warning notices and attempt to identify associated demographic risk characteristics. These results form the basis for our recommendations to improve counterfeit drug surveillance. Specifically, we recommend the dual use of statistical and geospatial methods to better identify demographic risk factors associated with counterfeit detections. These new methods can then translate to better information for all stakeholders involved and form the basis for enhanced prevention and reporting efforts. Efforts should be made to ensure that additional and validated data points are created through a multistakeholder counterfeit medicines surveillance model. Though counterfeit surveillance efforts are still in their infancy, ${ }^{34-36}$ we believe that statistical and geospatial methods can be helpful in improving detection and reporting of counterfeit medicines for Avastin and beyond. 
Author affiliations

${ }^{1}$ Global Health Policy Institute, La Jolla, California, USA

${ }^{2}$ Joint Doctoral Program in Global Public Health, University of California, San

Diego-San Diego State University, San Diego, California, USA

${ }^{3}$ Department of Anesthesiology, University of California, San Diego, California, USA

${ }^{4}$ Division of Global Public Health, University of California, San Diego, California, USA

Contributors TKM and REC conceived the study design. TKM gathered data on FDA notices, and REC gathered data on demographic variables. REC conducted statistical and geospatial analyses. TKM and REC provided interpretation of study findings.

Funding TKM is the recipient of an American Cancer Society Institutional Research Grant (70-002) provided through the Moores Cancer Center, UC San Diego that also provided support for REC and greatly acknowledge this support.

Competing interests None.

Ethics approval IRB approval was not required for this study.

Provenance and peer review Not commissioned; externally peer reviewed.

Data sharing statement No additional are data available.

Open Access This is an Open Access article distributed in accordance with the Creative Commons Attribution Non Commercial (CC BY-NC 4.0) license, which permits others to distribute, remix, adapt, build upon this work noncommercially, and license their derivative works on different terms, provided the original work is properly cited and the use is non-commercial. See: http:// creativecommons.org/licenses/by-nc/4.0/

\section{REFERENCES}

1. Siegel R, Ma J, Zou Z, et al. Cancer statistics, 2014. CA Cancer J Clin 2014;64:9-29.

2. Mackey TK, Liang BA. Oncology and the internet: regulatory failure and reform. J Oncol Pract 2012;8:341-3.

3. Kuehn BM. FDA warning: new batch of fake bevacizumab found $J$ Am Med Assoc 2013;309:864.

4. Kelesidis T, Kelesidis I, Rafailidis PI, et al. Counterfeit or substandard antimicrobial drugs: a review of the scientific evidence. $J$ Antimicrob Chemother 2007;60:214-36.

5. Food and Drug Administration. Counterfeit Version of Avastin in the U.S. Distribution. 2012

6. Eskens F, Sleifer S. The use of bevacizumab in colorectal, lung, breast, renal and ovarian cancer: where does it fit? Eur J Cancer 2008:44:2350-6.

7. Welch S, Spithoff K, Rumble RB, et al. Bevacizumab combined with chemotherapy for patients with advanced colorectal cancer: a systematic review. Ann Oncol 2010;21:1152-62.

8. Aghajanian C, Blank SV, Goff BA, et al. OCEANS: a randomized, double-blind, placebo-controlled phase III trial of chemotherapy with or without bevacizumab in patients with platinum-sensitive recurrent epithelial ovarian, primary peritoneal, or fallopian tube cancer. $J$ Clin Oncol 2012;30:2039-45.

9. Berenson A. A cancer drug shows promise, at a price that many can't pay. N Y Times 2006;15:2618-22.
10. FDA Center for Drug Evaluation and Research. Counterfeit version of Avastin in U.S. distribution. Silver Spring, Maryland. 2012.

11. Garcia-Aguirre G, Vanzinni-Zago V, Quiroz-Mercado H. Growth of Scytalidium sp. in a counterfeit bevacizumab bottle. Indian $J$ Ophthalmol 2013;61:523-5.

12. Weaver CM, Whalen J. How fake cancer drugs entered U.S. doctors' offices. Journal WS, 2012.

13. Nsimba SE. Problems associated with substandard and counterfeit drugs in developing countries: a review article on global implications of counterfeit drugs in the era of antiretroviral (ARVs) drugs in a free market economy. East Afr J Public Health 2008;5:205-10.

14. Liang BA. Parallel trade in pharmaceuticals: injecting the counterfeit element into the public health. $N C \mathrm{~J}$ Int Law Commercial Regul 2005;31:847-900.

15. Newton PN, Green MD, Fernández FM. Impact of poor-quality medicines in the "developing" world. Trends Pharmacol Sci 2010;31:99-101.

16. Fackler M. China's fake drugs kill thousands. San Francisco Examiner. 2002

17. Chakravarty S, Unnithan S, Ram A. Deadly doses. India Today. 2001.

18. Crampton T. Fatalities grow as people use cheap but worthless 'drugs': fake malaria pills haunt Asians. Times NY, 2003.

19. Mackey TK, Liang BA. The global counterfeit drug trade: patient safety and public health risks. J Pharm Sci 2011;100:4571-9.

20. Newton PN, Green MD, Fernández FM, et al. Counterfeit anti-infective drugs. Lancet Infect Dis 2006;6:602-13.

21. Food and Drug Administration. Health care provider alert: another counterfeit cancer medicine found in the United States. 2013

22. Cockburn R, Newton PN, Agyarko Ek, et al. The global threat of counterfeit drugs: why industry and government must communicate the dangers. PLoS Med 2005;4:e289.

23. Nelson M, Vizurraga M, Chang D. Counterfeit pharmaceuticals: A worldwide problem. The Trademark Reporter 1068. 2006.

24. Food and Drug Administration. Letters to doctors about risks of purchasing medications from foreign or unlicensed suppliers. Silver Spring, Maryland. 2012

25. U.S. Census Bureau. Maps and Data. Suitland, Maryland. 2014.

26. National Cancer Institute. Median Age of Cancer Patients at Diagnosisa, 2005-2009. Surveillance, Epidemiology and End-Results. Program. Rockville, Maryland. 2009.

27. U.S. Department of Labor. Labor force statistics from the current population survey. Washington DC: Statistics BoL, 2014.

28. Mukherji SK. Bevacizumab (Avastin). AJNR Am J Neuroradiol 2010;31:235-6.

29. Curran K. Cape Cod counterfeit drug ring focus of federal investigation. WCBV, 2013.

30. Partnership for Safe Medicines. Counterfeit cancer drugs are a big money maker for fake drug criminals. 2012.

31. Imber S. Update On Fake Avastin-FDA Warnings In 28 States, Six Prosecutions. Partnership for Safe Medicines. 2013.

32. Attaran A, Barry D, Basheer S, et al. How to achieve international action on falsified and substandard medicines. BMJ 2012;345:e7381.

33. Gostin LO, Buckley GJ, Kelley PW. Stemming the global trade in falsified and substandard medicines. JAMA 2013;309:1693-4.

34. Mackey TK, Liang BA. Improving global health governance to combat counterfeit medicines: a proposal for a UNODC-WHOInterpol trilateral mechanism. BMC Med 2013;11:233.

35. Institute of Medicine. Countering the problem of falsified and substandard drugs. 2013

36. WHO pilots surveillance system targeting fake meds. Reactions Weekly 1418:2. 2012 